Correlation of Students' Basic Understanding of Rigid Body Dynamics and Performance in Statics

Dr. Jeffrey L. Newcomer, Western Washington University 


\section{CORRELATION OF STUDENTS’ BASIC UNDERSTANDING OF RIGID BODY DYNAMICS AND PERFORMANCE IN STATICS}

\section{Introduction}

One of the most common specific difficulties that students display at the completion of a Statics class is the inability or unwillingness to consistently consider both force and moment equilibrium. This outcome has been clearly demonstrated by student performance on conceptual questions. $^{1-8}$ Too often students are either fixated on either force or moment equilibrium and apply only that one equilibrium condition, react to contextual cues and apply the condition that context invokes, or conflate force and moment equilibrium into a single condition. It would seem to stand to reason that a student who understands what would happen to a rigid body under load when it is not constrained to be static would have a better foundation upon which to build an understanding of rigid bodies under load that are constrained to be static, and therefore would show improved performance on traditional and conceptual statics problems.

The belief is that if a student has a faulty or inconsistent understanding of rigid body motion he or she will have more difficulty developing and consistently applying a correct approach to assessing static equilibrium. This faulty or inconsistent understanding could be labeled as a misconception, but the term 'misconception' is commonly used in engineering education to describe a strongly held incorrect understanding that must be unseated and replaced with a correct understanding. There is an alternative theory in Physics Education Research that student difficulties are influenced by context if not outright context dependent. ${ }^{9-15}$ In this view students' incoming understanding is made up of bits of loosely connected knowledge referred to as phenomenological primitives (p-prims) ${ }^{9-13}$ or facets of thinking ${ }^{14}$. These p-prims, or knowledge elements, are triggered by context; while they may individually be correct in some circumstances, they may be applied in the wrong circumstances or combined improperly to form incorrect conclusions. According to this view, students do not need to have misconceptions unseated; instead they must learn which elements are correct in which contexts. These elements provide building blocks for reaching the desired mode of thinking. In this terminology, instead of misconceptions, students exhibit "specific difficulties"15 - some more commonly than others. In order to design effective instructional material and techniques, one should take students' specific difficulties into account. $^{16-17}$ So in a sense the question here is: Do students who exhibit specific difficulties in describing rigid body motion at the beginning of Statics exhibit specific difficulties in assessing equilibrium at the beginning or end of Statics? If so, might identifying these students at the start of Statics allow the instructor(s) to more effectively address these students' expected challenges and give those students a better chance of exiting Statics with a consistent and correct approach to assessing equilibrium? This work attempts to answer the first of these two questions.

\section{Methodology}

To test the notion that understanding a dynamic rigid body gives a better foundation for understanding a static rigid body, students complete a pair of simple questions with an unconstrained rigid body on the first day of their Statics class before any material is covered. One question should be more likely to invoke only translation, and the other question should be more likely to 
invoke only rotation, but they are actually conceptually identical in effect in that both bars would begin to translate to the left and rotate counterclockwise, albeit with different accelerations. Figure 1 shows the two questions.

1. A bar is initially at rest and lying flat on a horizontal, frictionless table. The bar is then subject to the force acting at the location and direction shown.

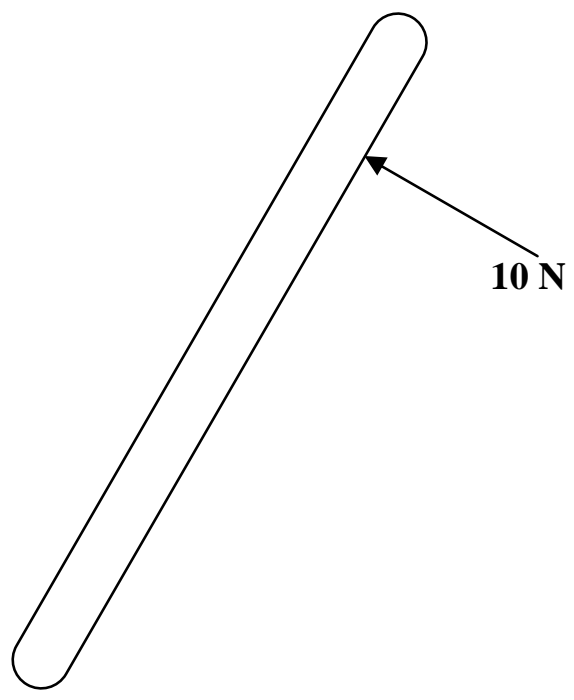

a) State whether the bar will continue to remain at rest once the force is applied.

b) If the bar does not remain at rest, then describe its subsequent motion as a result of the application of the force.
2. If the bar from problem 1 (initially at rest and lying flat on a horizontal, frictionless table) is subject to the two forces acting at the locations and directions shown.

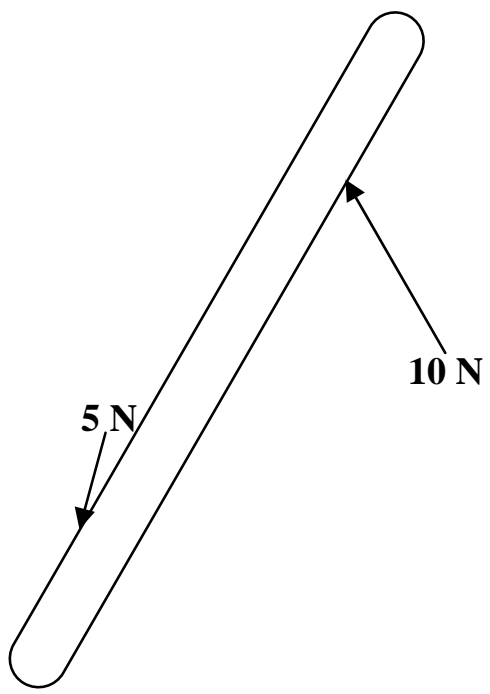

a) Explain how the bar's motion (or lack thereof) will differ from the situation in problem 1 , if at all.

Figure 1: Rigid body dynamics questions given the first day of Statics

Students' answers were analyzed to see whether students understood that the body would both translate and rotate in both cases, and coded accordingly. Performance on the rigid body motion questions were correlated to student performance on the Conceptual Assessment Tool for Statics (CATS) $^{*}$ pre-test and post-test results, both overall and on the specific equilibrium questions, and on performance on the final exam, both overall and on two conceptual questions, one on equilibrium and one on equivalence. In addition, entry data for each student was analyzed to determine if there were any correlations between past performance and performance on either the CATS pre-test or post-test, or on the final exam in Statics, both overall and on the conceptual questions. The specific entry data considered was: type of Physics I (calculus-based or algebrabased), location of Physics I (native or transfer), grade in Physics I, location of Calculus I (native or transfer), grade in Calculus I, whether Calculus II was taken before Statics or concurrently with it, the grade in Calculus II (if it had been completed), and overall GPA entering Statics. None of this entry data exhibited any statistically significant correlations with performance on either the CATS pre-test or post-test or on the final exam, on either traditional problems or conceptual questions.

\footnotetext{
${ }^{*}$ CATS was originally called the Statics Concept Inventory (SCI).
} 


\section{Analysis}

This study involved two different Statics classes at Western Washington University (WWU) during the spring 2011 and spring 2012 academic terms. The Statics course at WWU, which has a pre-requisite of one quarter of physics (mechanics) and two quarters of calculus (the second of which can be taken concurrently), is organized around five topics: free body diagrams, equilibrium, equivalence, separation of rigid bodies, and friction, without differentiation between two vs. three dimensional cases, concurrent vs. non-concurrent force systems, and single bodies vs. frames and trusses. ${ }^{18}$ These situations are all addressed in the course, but not in the order of traditional textbooks. The course does not require a text, but in place of a text the students all registered for the Open Learning Initiative (OLI) Statics. ${ }^{19}$ The only difference between the two sections was that in spring 2011 use of OLI Statics was strongly encouraged, but optional, but in spring 2012 eight of the OLI Statics quizzes were assigned as homework problems, which also provided some interesting information relative to this project for that group of students. Otherwise the course is a standard lecture-based course with homework, midterms, projects, a final exam, a limited number of think-pair-share exercises, and weekly Warm Up (WU) exercises for the first eight weeks of the quarter. ${ }^{20}$ The WU exercises are all based upon CATS questions, so they help to maintain an emphasis on conceptual issues in addition to problem solving technique.

A total of 96 students registered for these two sections, but four did not attend the first day of class, so the study is based upon 92 responses to the questions shown in Figure 1 and subsequent work done by those students. Each response to both questions was scored with a 1 or 0 for both translation and rotation to indicate whether the response acknowledged that each bar would translate or not and rotate or not respectively. Students were then coded based upon the four scores. The coding scheme is as follows:

All (A) - Students acknowledged that both bars would both translate and rotate. This grouping contained 36 students (39\% of respondents).

Expected Pattern (EP) - Students identified the first bar as translating but not rotating, and the second bar as rotating but not translating. This grouping contained 10 students (11\% of respondents).

Translation only ( $\mathrm{T})$ - Students identified both bars as translating and neither as rotating. This grouping contained 8 students ( $9 \%$ of respondents).

Rotation only (R) - Students identified both bars as rotating and neither as translating. This grouping contained 16 students (17\% of respondents).

Almost All/Expected Pattern (A/E) - Students either identified the first bar as only translating and the second bar as translating and rotating, or they identified the first bar as translating and rotating and the second bar as only rotating. This grouping contained 13 students (14\% of respondents).

Other (O) - Students responses did not follow a pattern that fit into any of the other categories. This grouping contained 9 students ( $10 \%$ of respondents). 
The first four groupings are self-evident, but the last two deserve a bit more explanation. The original coding scheme did not contain the A/E grouping, but it became clear during the coding process that there was a certain logic to that set of responses in that they fell into the expected pattern for one of the two bars and gave a correct explanation for the other, so those students were separated out from the remaining students in the $\mathrm{O}$ grouping. The students in the O grouping did not show any consistent pattern in their responses and include three cases where students claimed that one or both of the bars would not move at all.

Entry data for each of the coded groupings was compared. While overall GPA was effectively the same for all grouping, students in the $\mathrm{O}$ grouping achieved lower grades in Physics I and Calculus I, the latter of which was statistically significant relative to the other grouping $(\mathrm{p}=$ 0.064). Students in the $O$ grouping were also more likely to have taken algebra-based rather than calculus-based Physics, which was not true for any of the other grouping. Otherwise the entry data for the six grouping was statistically indistinguishable. Another area that was statistically indistinguishable between the six grouping was students' scores on the CATS pre-test that they took the end of the first week of Statics, for both their overall scores and their scores on the equilibrium section of CATS.

Performance at the end of Statics showed slightly more correlation with the coded groupings of responses on the rigid body dynamics question than the entry data. Five aspects of students' performance at the end of Statics were compared to the coded groupings on the rigid body dynamics question: 1) Overall performance on the final exam, which included five traditional questions and two conceptual questions; 2) performance on the equilibrium conceptual question on the final exam, which is based upon question 26 on CATS; 3) performance on the equivalence conceptual question on the final exam, which is based upon question 8 on CATS; 4) performance on the CATS post-test; and 5) performance on the equilibrium questions, questions 25-27, on the CATS post-test.

There were no discernable, much less statistically significant, differences between students in the A, A/E, R, and T groupings for any of the five areas, so those students were grouped together for comparisons with the other groupings. In all five cases students in the EP category had slightly lower average performance than students in the A, A/E, R, and T categories, but only the difference on the overall CATS post-test was statistically significant $(\mathrm{p}=0.063)$. In all five cases students in the $\mathrm{O}$ category had the lowest average performance, and four of those differences were statistically significant when compared to students in the A, A/E, R, and T categories: performance on the equilibrium conceptual question on the final exam $(p=0.082)$, performance on the equivalence conceptual question on the final exam $(p<0.002)$, overall performance on the CATS post-test $(\mathrm{p}=0.004)$, and performance on the equilibrium section of the CATS post-test ( $\mathrm{p}$ $=0.004$ ). In other words, students whose responses on the rigid body dynamics question at the start of Statics placed them into the O grouping showed notably worse performance on all conceptual measures at the end of the Statics course when compared to students in the $A, A / E, R$, and $\mathrm{T}$ groupings.

Further evidence of the conceptual divide between students in the different groupings can be seen by comparing the CATS pre-test and post-test scores. The overall average on the pre-test was 10.0 out of 27 with the groupings ranging from a high average of 10.7 points (EP) to a low 
average of 8.8 points (R). On the post-test students in the A, A/E, R, and T groupings had an average increase of 7.8 points, students in the EP grouping had an average increase of 4.9 points, but students in the $\mathrm{O}$ grouping had an average increase of only 0.4 points.

Students in the spring 2012 section of Statics had an additional chance to answer rigid body dynamics questions on an OLI Statics quiz on the Effects of Force. Of the 50 students in the spring 2012 section who completed the in-class rigid body dynamics questions shown in Figure 1, 44 of them also completed this OLI Statics quiz. One of the questions on the OLI Statics quiz was identical to problem 1 in Figure 1 (except for orientation). For that problem $79 \%$ of students in the $\mathrm{A}, \mathrm{A} / \mathrm{E}$, and $\mathrm{T}$ groupings correctly identified the bar as both rotating and translating, while only $60 \%$ of students in the R, EP, and O groupings did so. Interestingly however, when the force was moved to either the end or middle of the bar and applied perpendicular to the bar all students in all categories correctly identified the motion that would result. As part of the same quiz, students in the R, T, EP, and O groupings had more difficulty correctly identifying two bars with the same translational velocity (50\% successful) than students in the A and A/E groupings (75\% successful), and students in the EP and O groupings had more difficulty correctly identifying two bars with the same rotational velocity (43\% successful) than students in the A, A/E, R, and $\mathrm{T}$ groupings ( $76 \%$ successful). While none of these results are statistically significant due to the small numbers of students involved, they provide further indication that students in the EP and $\mathrm{O}$ groupings struggle more with conceptual issues related to forces and Statics than students in the other groupings.

\section{Conclusions}

The question that motivated this study was whether the ability or inability to correctly describe rigid body motion would correlate to an ability or inability to correctly assess static equilibrium. While the data set is relatively small, the results to this point indicate that students' explanations to rigid body dynamics questions can be correlated to their exhibiting difficulty assessing static equilibrium, as well as other concepts in Statics, but only for a very limited set of students.

According to the data, the important thing was not that students answered both rigid body dynamics questions correctly but that they exhibited a consistency in their approach to those problems. Those students who got both questions right, got one right and followed an expected pattern on the other, or consistently assigned only translation or rotation and never the other showed consistent performance and improvement in Statics on both traditional and conceptual problems. In contrast, those students who alternated between translation and rotation, potentially based on there being one force or two, showed consistently lower, but not significantly lower, performance and improvement in all aspects of Statics at the end of the class. Most strikingly, those students who did not exhibit a consistent approach in their answers to the rigid body dynamics questions exhibited poorer performance in all aspects of Statics at the end of the class. Moreover, their performance on conceptual aspects of Statics was significantly lower than other students, and they showed almost no improvement in the conceptual aspects of the class despite the emphasis placed on that area through the Warm Up exercises. These findings are consistent with studies of students' explanations to an equilibrium question (question 26 on CATS) in that students who inconsistently apply force or moment equilibrium, but not both, have a weaker con-

ceptual foundation than those who consistently apply one or the other (but not both). ${ }^{3,46}$ Neither 
group is assessing equilibrium correctly, but the latter group is at least doing an aspect correctly, while the former group appears to continue to conflate force and moment equilibrium into one condition.

The future work for this project is to confirm the results and determine if it is possible to work with students whose answers to the rigid body dynamics questions are inconsistent to help them strengthen their conceptual understanding of Statics (and maybe the underlying Physics), because it is clear that while the course structure works for the majority of students, it is not working for these students. There was a hope at the start of this project that students would exhibit consistency between assessing rigid body dynamics and static equilibrium in ways such as students who assume that the rigid bodies would only translate in the dynamic situation would be more likely to assess only force equilibrium in the static situation, but that was not borne out by the data. So while the results are more narrow than originally hoped for, the correlation between showing inconsistency on rigid body dynamics problems and struggling with conceptual problems in Statics does seem to indicate that some students need more scaffolding in order to grasp the fundamental concepts of force and moment, and that it might be relatively easy to identify these students early in a course and find ways to improve their conceptual understanding.

\section{Acknowledgements}

This work was supported in part by the National Science Foundation through grant DUE 0918271.

\section{Bibliography}

1. Ortiz, L. G., Heron, P. R. L., and Shaffer, P. S., "Student Understanding of Statics Equilibrium: Predicting and Accounting for Balancing,” American Journal of Physics, Vol. 73, No. 6, 2005, pp. 545-553.

2. Newcomer, J. L., and Steif, P. S., "Student Explanation of Answers as a Window into Prior Misconceptions," Proceedings of the 36th Annual ASEE/IEE Frontiers in Education Conference, San Diego, CA, Oct. 2006, pp. S4H-6-11.

3. Newcomer, J. L., and Steif, P. S., "Gaining Insight into Student Thinking from their Explanations of a Concept Question," Proceedings of the 1st International Conference on Research in Engineering Education, Honolulu, HI, June 2007, pp. 1-8.

4. Newcomer, J. L., and Steif, P. S., "Testing The Commonality Of Student Conceptual Explanations Across Institutions," Proceedings of the 2007 ASME International Mechanical Engineering Congress and Exposition, Seattle, WA, Nov. 2007, pp. 1-9.

5. Newcomer, J. L., and Steif, P. S., "What Students 'Know' About Statics: Specific Difficulties Common Among Students Entering Statics," Proceedings of the 38th Annual ASEE/IEE Frontiers in Education Conference, Saratoga Springs, NY, Oct. 2008, pp. S1C-1-6.

6. Newcomer, J. L., and Steif, P. S., "Student Thinking about Static Equilibrium: Insights from Written Explanations to a Concept Question,” Journal of Engineering Education, Vol. 97, No. 4, pp. 481-490. 
7. Newcomer, J. L., "Inconsistencies in Students' Approaches to Solving Problems in Engineering Statics," Proceedings of the 40th Annual ASEE/IEE Frontiers in Education Conference, Washington, DC, Oct. 2010, pp. F3G-1-6.

8. Brose, A., and Kautz, C. H., "Identifying and Addressing Student Difficulties in Engineering Statics," Proceedings of the 2011 ASEE Annual Conference and Exposition, Vancouver, BC, June 2011.

9. diSessa, A., "Towards an Epistemology of Physics," Cognition and Instruction, Vol. 10, Nos. 2 and 3, 1993, pp. 105-225.

10. Hammer, D., "Misconceptions Or P-Prims: How May Alternative Perspectives Of Cognitive Structure Influence Instructional Perceptions And Intentions,” The Journal of the Learning Sciences, Vol. 5, No. 2, 1996, pp. 97-127.

11. Hammer, D., "More Than Misconceptions: Multiple Perspectives On Student Knowledge And Reasoning, And An Appropriate Role For Education Research,” American Journal of Physics, Vol. 64, No. 10, 1996, pp. 1316-1325.

12. Elby, A., "What Students' Learning Of Representations Tells Us About Constructivism," Journal of Mathematical Behavior, Vol. 19, No. 4, 2000, pp. 481-502.

13. Redish, E. F., "A Theoretical Framework For Physics Education Research: Modeling Student Thinking,” Proceedings of the International School of Physics "Enrico Fermi" Course CLVI, Varenna, Italy, E.F. Redish and M. Vicentini (Eds.), Amsterdam: IOS Press, 2004, pp. 1-50.

14. Minstrell, J, "Facets Of Students' Knowledge And Relevant Instruction," Proceedings of an International Workshop - Research in Physics Learning: Theoretical Issues and Empirical Studies. R. Duit, F. Goldberg, and H. Niedderer (Eds.), Kiel, Germany: The Institute for Science Education (IPN), 1992, pp. 110128.

15. Loverude, M. E., Kautz, C. H., and Heron, P. R. L., "Student Understanding Of The First Law Of Thermodynamics: Relating Work To The Adiabatic Compression Of An Ideal Gas,” American Journal of Physics, Vol. 70, No. 2, 2002, pp. 137-148.

16. Heron, P. R. L., "Empirical Investigations Of Learning And Teaching, Part I: Examining And Interpreting Student Thinking,' Proceedings of the International School of Physics "Enrico Fermi" Course CLVI, Varenna, Italy, E. F. Redish and M. Vicentini (Eds.), Amsterdam: IOS Press, 2004, pp. 341-350.

17. Heron, P. R. L., "Empirical Investigations Of Learning And Teaching, Part II: Developing Research-Based Instructional Materials,' Proceedings of the International School of Physics "Enrico Fermi" Course CLVI, Varenna, Italy, E. F. Redish and M. Vicentini (Eds.), Amsterdam: IOS Press, 2004, pp. 351-365.

18. Newcomer, J. L., "Many Problems, One Solution Method: Teaching Statics without 'Special Cases'," Proceedings of the 36th Annual ASEE/IEE Frontiers in Education Conference, San Diego, CA, Oct. 2006, pp. S2D-7-12.

19. Dollár, A., and Steif, P. S., “An Interactive, Cognitively Informed, Web-based Statics Course,” International Journal of Engineering Education, Vol. 24, No. 6, pp. 1229-1241, 2008

OLI Statics web site: https://oli.cmu.edu/jcourse/webui/guest/join.do?section=statics

20. Patterson, E., and Novak, G., Just-In-Time Teaching, http://webphysics.iupui.edu/jitt/jitt.html. 